ICAASET-2021, 20-21 May, 2021, K.R. Mangalam University, Gurugram

International Journal of Technical Research \& Science (Special Issue) ISSN No.:2454-2024 (online)

\title{
REVIEWING THE PID BASED TECHNOLOGIES BEHIND TWO WHEEL BALANCING ROBOT
}

\author{
Shubham Malpani' ${ }^{1}$, Shrishti Gupta ${ }^{2}$, Shreyanshi Agarwal ${ }^{3}$, Mohd. Alam ${ }^{4}$ \\ Geetanjali Raj \\ Email Id: shubham.17bec1031@abes.ac.in, shrishti.17bec1128@abes.ac.in, \\ shreyanshi.17bec1146@abes.ac.in, mohd.17bec1194@abes.ac.in, geetanjali.raj@abes.ac.in \\ ABES Engineering College, Ghaziabad, Uttar Pradesh, India
}

\begin{abstract}
The analysis of the concept for self-balancing robots and its theory and implementations was proposed in this paper. Self-balancing bots are based on the inverted pendulum theory. In crisis situations and mountain regions, these types of bots can be used to provide medicines. A Self Balanced Robot has the ability to stand upright only by using the two wheels that minimise the bot 's height. As a Spy Bot, it has a really successful application. It relies essentially on the inverted framework of pendulum and balancing with sophisticated controllers and PID algorithms. The idea of creating this self-balancing bot is an inspiration for potentially moving two-wheel bots that can make it simpler and more effective for a man to operate. We can reduce the risk of human life mortality by using bots in hilly areas, such as mountains, in the event of disasters to provide services and to provide rescue operations at times of need.
\end{abstract}

Keywords: PID-Proportional-Integral-Derivative Controller, Inverted Pendulum, MATLAB Design, MPU-6050.

\section{INTRODUCTION}

This study paper allows one to understand the creation of self-balancing robots with two independently driven wheels and a centre of gravity above the axis of rotation of the wheels. The robots' motion approaches the pendulum inverted. The device has a remote control for the user to monitor the movement of the robot. We can display video images in real-time using the MATLAB GUI. The MPU-6050 sensor is used to calculate the tilt of the robot.MPU-6050 is the sensor for calculating robot inclination.[5] These robots have made rapid strides or have been in many fields of public concern and their everyday lives over the past decade. There are also some models that can help people in daily life, such as travel aids, etc., which are based on the above mentioned model. These robots are based on the wide variety of applications, including their lightweight nature and tight power with a null turning radius[5].
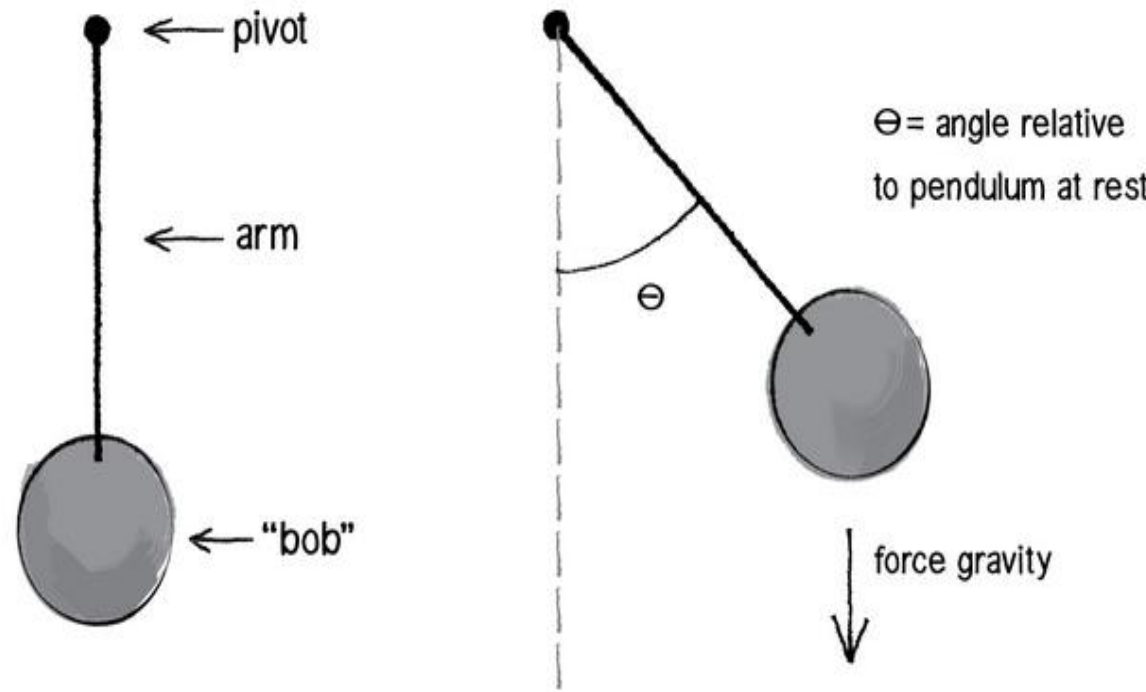

Fig. 1.1 Concept of Simple Pendulum, The force of gravity is in downward direction. The component of this force is responsible to make bob in a stable position.

\section{LITERATURE REVIEW}

This section will include perspectives and literature reviews on the latest technologies and methodologies that are accessible and used to build a two-wheel self-balancing robot. The fundamental concept behind the equilibrating robot is based on a pendulum model and has been widely used worldwide in developing wheel robotics along with other robotic types including legged robots.[12] A double wheeled robot design is introduced using the Digital Signal Processor based on researchers in the Industrial Electronics Laboratory of the German Federal Institute of Technology. A linear state space controller using gyroscope and engine encoder sensors is used to balance this device[5]. 
ICAASET-2021, 20-21 May, 2020, K.R. Mangalam University, Gurugram

International Journal of Technical Research \& Science (Special Issue) ISSN No.:2454-2024 (online)

\subsection{Previous project works}

Dean Kamen, who has developed a lot of control systems, has created a two-wheeled robot called SEGWAY HT.

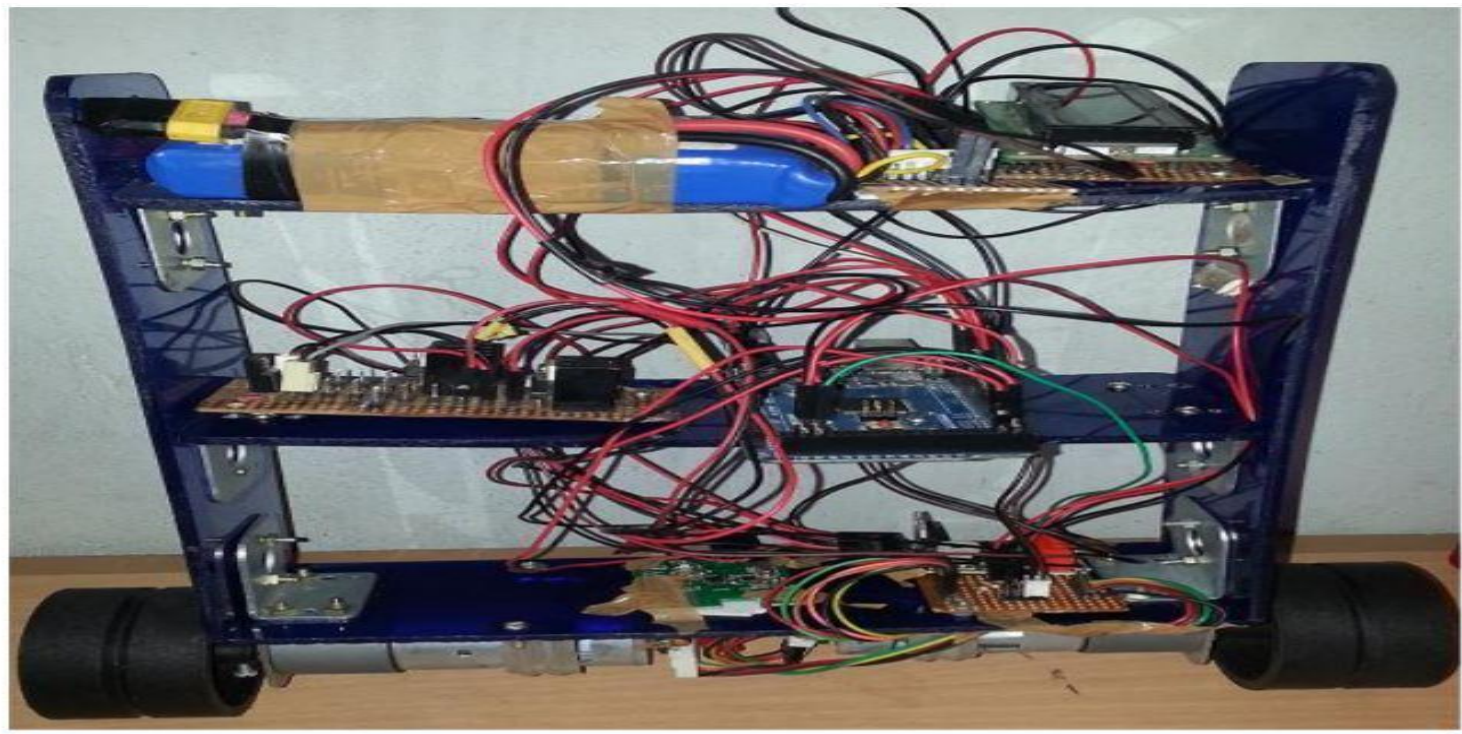

Fig. 2.1 Previous Model of SBB(Self Balancing Bot)

Although this robot has an additional function, while a user stands on top of it and navigates the ground with it, it can balance it. A two-wheeled robot with digital signal processor control is also available for another robot built by researchers from the Industrial Electronics laboratory of the Swiss Federal Institute of Technology. Steve Hassenplug produced a self balancing robot with a more innovative approach. The Nbot, designed by David. P.Anderson.Some people at Columbia University still have Brennan's Monorail releases and modifications. But the group was sadly unable to develop a working model. During use, the electronic component of the model was overheated and the motor was burnt. The electronic section was not well and did not perform well [9].

The figure above demonstrates the working model of a self balancing bot.

\subsection{Control system}

After all, there are only two types of controls used to govern a system that is known as linear and non-linear controls. Proportional, integral and derivative, commonly known as PID, is one of the most famous controls used by many researchers. A number of variables required to be considered in order to create the framework accordingly applied. So, the system is complex. Nonetheless, few requirements required to model the system are smaller in scale. Because of this, it is preferred by most of the researchers because it is simpler and effective in some instances[19].

\section{TWO WHEEL BALANCING CONTROL TECHNOLOGIES}

In order to sustain a balanced robot, the motors have to counter the robot collapse, and input and element correction are important for this step. The MPU6050 gyroscope and accelerometer feedback element is used to accelerate and rotate all three axes. Arduino is used to know the current robot orientation of the robot. The MPU6050 sensor module is a full motion detector with a three-axis gyroscope, a 3-axes accelerometer and a digital motion processor made up of an entirely small box. It is also fitted with a sensor of temperature. It has an I2C bus protocol for communicating with microcontrollers[11]. The 3-axis MPU6050 gyroscope tests the angular rate (turning speed) around the three axes. In order to be able to calculate the rate of fall of the robot, the cornering speed on the $x$ axis is sufficient for our self-balancing robot alone. We made two measurements from two different sources. The calculation of the accelerometer is influenced by abrupt horizontal motions and the calculation of the gyroscope steadily changes away from the actual meaning. In MPU6050, short-duration signals are used to read the accelerometer and long-duration signals have an effect on the reading of the gyroscope.[8] 
ICAASET-2021, 20-21 May, 2020, K.R. Mangalam University, Gurugram

International Journal of Technical Research \& Science (Special Issue) ISSN No.:2454-2024 (online)

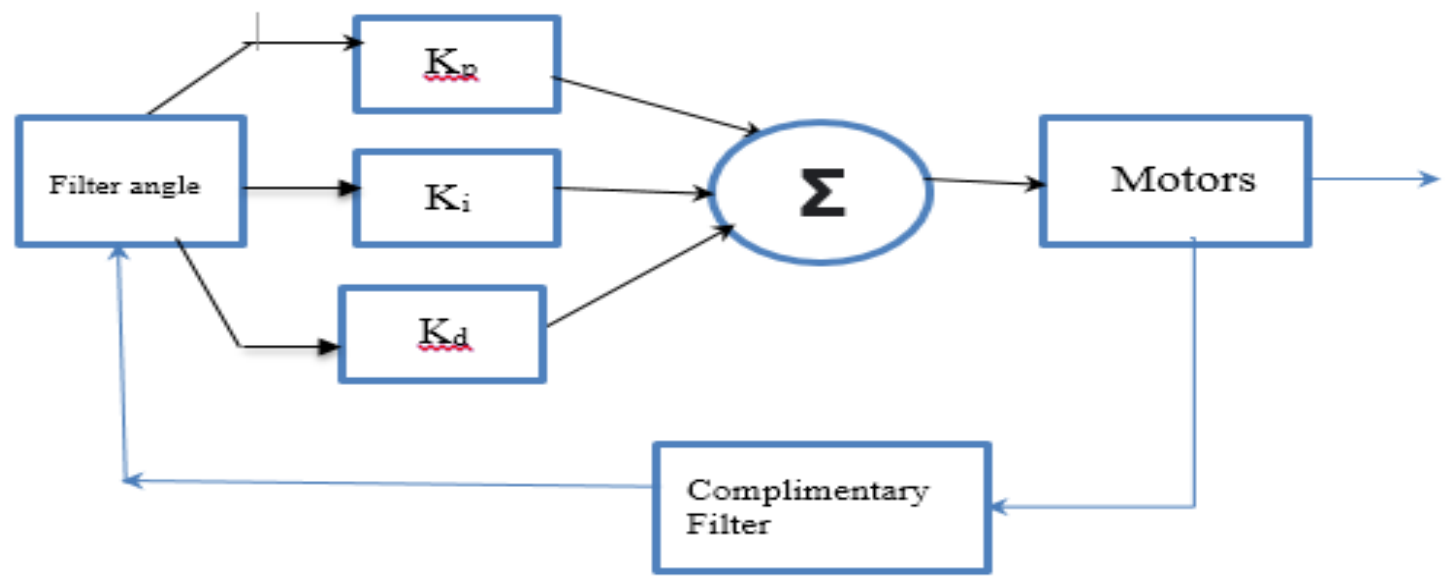

Fig. 3.1 PID Control Parameters, kp,ki and kd..is used for removing steady state errors, reducing oscillation and making them stable[20]

The accelerometer and gyroscope readings are complementary to each other. To get a secure, precise measurement of the angle, we combine them both using a Complementary Filter. The filter is essentially a high-pass gyroscope filter and a low-pass filter with the effect of the accelerometer to filter out drift and noise from the measurement. The controller to control the inclination angle is a PID controller, a common industrial automation controller. To attach a camera to tape the image, the slab and tilt platform must be installed and placed on top of the robot[3]. A remote controller that controls the movement of the robot as well as the pan and robot tilt is included in the device.A MATLAB Interface needs to be developed to allow the user to track video in real-time and to save a video on a hard disc. This paper will discuss various existing approaches for implementing the scheme[10].

\subsection{Self Balancing Algorithm}

We suggested mixing accelerometer and gyroscope sensor readings in the study paper to calculate the angle of inclination in the development of the double-wheel self-evolution robot based on the algorithm of particle swarm optimization. In the right inclination angle are using both the accelerometer and the gyroscope. Both are used in the MPU6050. We receive all accelerometers and gyroscope data and then fuse the readings with either the Kalman Filter or the use of complementary Filter. The research document further states that the investigator has attempted to eliminate noise from the sensor of the MPU-6050 with the Kalman filter and the additional filter, which has been shown to be swift and stable when opposed to heavy Kalman filters. The use of the supplementary filter would be much simpler.The paper also shows the use of PID controllers to enhance stability in the robot.[22] The research paper contrasts both the linear and the PID controllers and finds that, in terms of efficiency, the LQR is much better than the PID. With the help of a computer-aided design programme, The model is created for the wheeled self-balancing robot and then exported to MATLAB / Simmechanics, which is a MATLAB multibody modelling toolbox. The LQR controller is developed and applied to the model using MATLAB / Simulink software after linearization. A PID controller is the main controller used to build this self-balanced robot and complementary filter is the filter used[7].

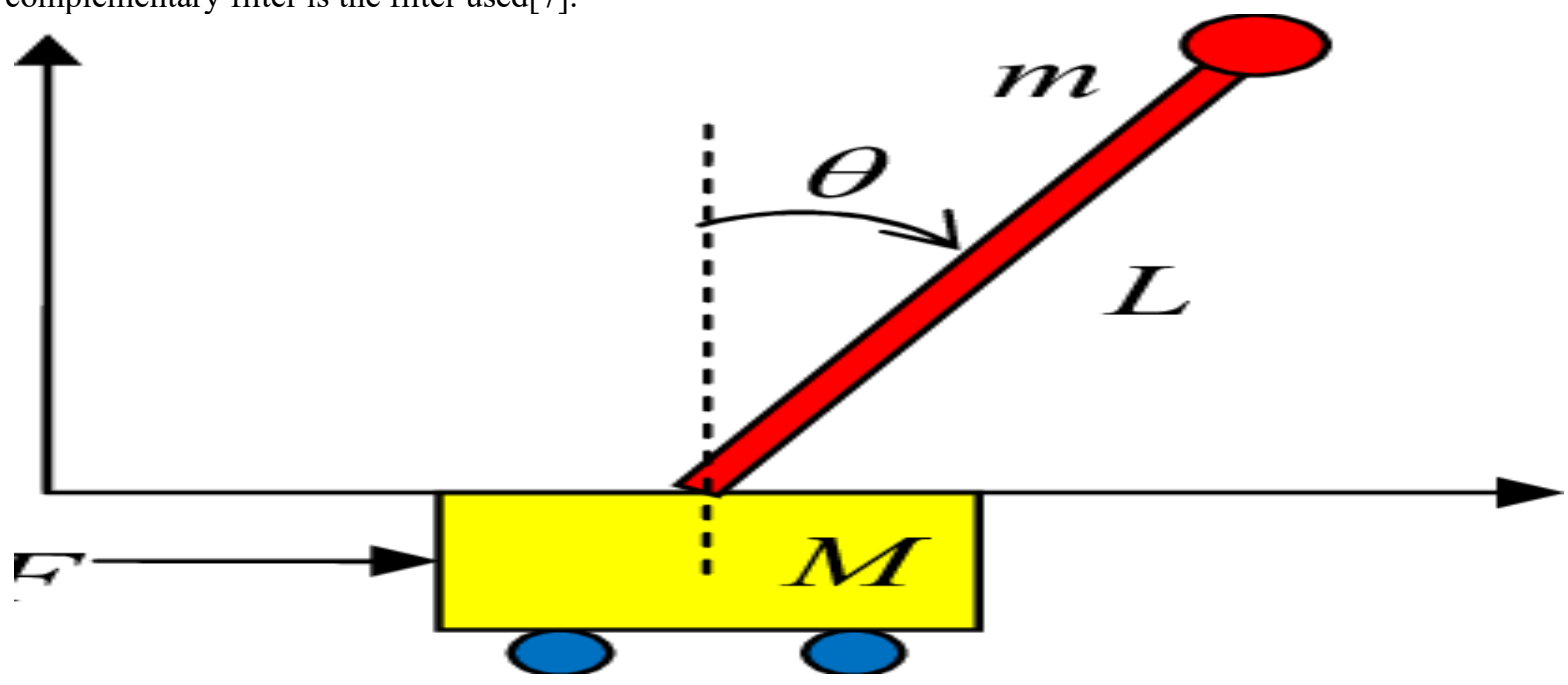

Fig. 3.2 Model of Inverted Pendulum

According to the report, the project succeeded with robot stability being satisfactory. The foundations behind the auto balance bot control mechanism are the stability of the inverted pendulum by using strength at its hinge.The paper describes another method for PID controller design whereby furry adaptive gains should be allocated to PID controllers rather than constant PID gains for each inclination value. High stability and low engine noise efficiency have the effect of such an implementation. 
ICAASET-2021, 20-21 May, 2020, K.R. Mangalam University, Gurugram

International Journal of Technical Research \& Science (Special Issue) ISSN No.:2454-2024 (online)

Let's take an example of a two-dimensional device in which the pendulum is confined to a vertical plane. The mechanism consists of experimental components to drive the cart horizontally, such as control input Force F, and the outputs are theta, which is the angular position of the pendulum and distance ' $\mathrm{X}$ ' from the origin. The figure (4) above represents a cart pendulum system with all the experimental components and its specifications.

\subsection{Modeling of Non-Linear Dynamical System}

In mathematics and science, a non-linear system is a system in which the output change is not linearly proportional to the input change. Non-linear problems are of interest to engineers, scientists, mathematicians, etc. since basically, most natural processes are non-linear. In contrast to far simpler linear systems, non-linear dynamic systems that reflect changes in variables over time frequently appear chaotic, unpredictable, or counter-intuitive in nature. A nonlinear system usually defines its behavior as a set of concurrent equations in which unknowns appear as polynomial variables of a higher degree than one (or unknown functions for different equations). This method is considered a non-linear method of equations[5].

\section{CONCLUSION}

In this paper, we focused on evaluating and experimenting on two wheels with the aim of moving away from selfbalancing, navigation or avoiding obstacles in order to be able to perform tasks such as transporting or monitoring the surrounding area. Improved methods of control and artificial intelligence have been streamlined in order to maintain balance control.

The system is effectively operated by linear controls, non-linear controllers and self-adapting controllers.

The experiment would have to be assessed on the basis of trajectory, speed, speed setting, obstacle avoidance technique and contact with the surrounding environment.

\section{FUTURE SCOPE}

Self-balancing bots can be used to provide medicines in situations of pandemic. Highly useful for medical assistance in mountain areas where humans find it difficult to access. In the future, several more innovations will be implemented and will be of assistance in the area of robotics.

Modern vehicles such as the Segway or Hover board and Ball Bot are the most popular examples of this robotic platform. In their everyday lives, more ideas will help individuals to use bots.

\section{REFERENCES}

[1] Çakan, Abdullah \& Botsali, Fatih. (2016). Modeling and LQR Control of a Wheeled Self-Balancing Robot.

[2] Kumar, C. \& Lal, S. \& Patra, Nilanjan \& Halder, Kaushik \& Reza, Motahar. (2012). Optimal controller design for inverted pendulum system based on LQR method. 259-263. 10.1109/ICACCCT.2012.6320782.

[3] Romlay, Muhammad Rabani \& Mohd Ibrahim, Siti \& Rashid, Azhar \& Toha, Mahbub. (2019). Two-wheel Balancing Robot; Methods and experiments of control analysis. International Journal of Modern Engineering and Technology. 7.

[4] ISSN: 2455-6211, Volume 6, Issue 9, September-2018, Impact Factor: 2.287. Vishnu Diwakar, Anuj Gupta, UG Student, Dept. of Mechatronics, Asia Pacific Institute of Information Technology, Study Paper on SelfBalancing Two-Wheel Spy Robot, India, International Journal of All Science Education and Scientific Methods (IJARESM)

[5] Frankovský, P., Dominik, L., Gmiterko, A., Virgala, I., Kurylo, P., \& Perminova, O. (2017). Modeling of two-wheeled self-balancing robots driven by DC gearmotors. International Journal of Applied Mechanics and Engineering, 22(3), 739-747.

[6] Pratyusha Kumar Tripathy, under Prof, S.K. Das, UG Student, Electronics and Communication Engineering, National Institute of Technology.

[7] Mateşică, Iulian, Mihai Nicolae, Liliana Bărbulescu, and Ana-Maria Mărgeruşeanu. "Self-balancing robot implementing the inverted pendulum concept." In 2016 15th RoEduNet Conference: Networking in Education and Research, pp. 1-5. IEEE, 2016.

[8] Ahmadi Jeyed, Hamze and Ghaffari, Ali, 2020, "A nonlinear optimal control based on the SDRE technique for the two-wheeled self-balancing robot" Australian Journal of Mechanical Engineering pp 1, 2204-2253

[9] Wang, Chundong, Jin Huang, Chao Wang, and Rui Wu. "Dynamic Surface Active Disturbance Rejection Control for Two-Wheeled Self-Balancing Robot." In Proceedings of the 3rd International Conference on Robotics, Control and Automation, pp. 82-87. 2018

[10] G. Madhumitha, R. Srividhya, J. Johnson and D. Annamalai, "Physical modeling and control of self-balancing platform on a cart," 2016 International Conference on Robotics: Current Trends and Future Challenges (RCTFC), Thanjavur, 2016, pp. 1-6, doi: 10.1109/RCTFC.2016.7893410.

[11] H. Juang and K. Lurrr, "Design and control of a two-wheel self-balancing robot using the arduino microcontroller board," 2013 10th IEEE International Conference on Control and Automation (ICCA), Hangzhou, 2013, pp. 634-639, doi: 10.1109/ICCA.2013.6565146.

[12] L. Sun and J. Gan, "Researching of Two-Wheeled Self-Balancing Robot Base on LQR Combined with PID," 2010 2nd International Workshop on Intelligent Systems and Applications, Wuhan, 2010, pp. 1-5, doi: 10.1109/IWISA.2010.5473610.

[13] Nguyen Gia Minh Thao, Duong Hoai Nghia and Nguyen Huu Phuc, "A PID backstepping controller for twowheeled self-balancing robot," International Forum on Strategic Technology 2010, Ulsan, 2010, pp. 76-81, doi: 10.1109/IFOST.2010.5668001.

DOI Number: https://doi.org/10.30780/specialissue-ICAASET021/011 
ICAASET-2021, 20-21 May, 2020, K.R. Mangalam University, Gurugram

International Journal of Technical Research \& Science (Special Issue) ISSN No.:2454-2024 (online)

[14] W. An and Y. Li, "Simulation and control of a two-wheeled self-balancing robot," 2013 IEEE International Conference on Robotics and Biomimetics (ROBIO), Shenzhen, 2013, pp. 456-461, doi: 10.1109/ROBIO.2013.6739501.

[15] Junfeng Wu, Yuxin Liang and Zhe Wang, "A robust control method of two-wheeled self-balancing robot," Proceedings of 2011 6th International Forum on Strategic Technology, Harbin, Heilongjiang, 2011, pp. 10311035, doi: 10.1109/IFOST.2011.6021196.

[16] F. Sun, Z. Yu and H. Yang, "A design for two-wheeled self-balancing robot based on Kalman filter and LQR," 2014 International Conference on Mechatronics and Control (ICMC), Jinzhou, 2014, pp. 612-616, doi: 10.1109/ICMC.2014.7231628.

[17] B. Mahler and J. Haase, "Mathematical model and control strategy of a two-wheeled self-balancing robot," IECON 2013 - 39th Annual Conference of the IEEE Industrial Electronics Society, Vienna, 2013, pp. 41984203, doi: 10.1109/IECON.2013.6699809.

[18] K. Prakash and K. Thomas, "Study of controllers for a two wheeled self-balancing robot," 2016 International Conference on Next Generation Intelligent Systems (ICNGIS), Kottayam, 2016, pp. 1-7, doi: 10.1109/ICNGIS.2016.7854009.

[19] R. S. Martins and F. Nunes, "Control system for a self-balancing robot," 2017 4th Experiment@International Conference (exp.at'17), Faro, 2017, pp. 297-302, doi: 10.1109/EXPAT.2017.7984388.

[20] M. Engin, "Embedded LQR controller design for self-balancing robot," 2018 7th Mediterranean Conference on Embedded Computing (MECO), Budva, 2018, pp. 1-4, doi: 10.1109/MECO.2018.8405959.

[21] Bhagat, Siddhesh R.. "Self Balancing Robot." International Journal for Research in Applied Science and Engineering Technology 6 (2018): 180-184.

[22] Arani, Mikail S., et al. "Sliding Mode Control Design of a Two-Wheel Inverted Pendulum Robot: Simulation, Design." Advances in Motion Sensing and Control for Robotic Applications: Selected Papers from the Symposium on Mechatronics, Robotics, and Control (SMRC'18)-CSME International Congress 2018, May 27-30, 2018 Toronto, Canada. Springer, 2019.

[23] I. Mateşică, L. Bărbulescu, M. Nicolae, and A. Mărgeruşeanu, " Self-balancing robot implementing the inverted pendulum concept" 2016 15th RoEduNet Conference: Education and Science Networking, Bucharest, 2016, pp. 1-5, doi: 10.1109 / RoEduNet.2016.7753230. 2016.

[24] S. Sarathy, M. M. Mariyam Hibah, S. Anusooya and S. Kalaivani, "Implementation of Efficient Self Balancing Robot," 2018 International Conference on Recent Trends in Electrical, Control and Communication (RTECC), Malaysia, Malaysia, 2018, pp. 65-70, doi: 10.1109/RTECC.2018.8625624.

[25] Sahil Patil, Dhananjay Bhavsar, Shubham Mantri, Shraddha Gosavi, 2021, Self Balancing Robot, INTERNATIONAL JOURNAL OF ENGINEERING RESEARCH \& TECHNOLOGY (IJERT) NTASU 2020 (Volume 09 - Issue 03),

[26] Michahial, Stafford \& Basavanna, Mr \& Mahadevappa, Shivakumar. (2015). Two Wheeled Balancing Autonomous Robots. International Journal of Advanced Research in Computer and Communication Engineering. 4. 10.17148/IJARCCE.2015.41128. 